\title{
Institutions, economic openness and credit cycles: An international evidence
}

\section{Canh Phuc Nguyen}

School of Banking, University of Economics Ho Chi Minh City,

Vietnam

Canbnguyen@ueh.edu.vn

\section{Christophe Schinckus}

School of Accounting and Finance, Taylor's University,

Malaysia

Christophe.scbinck.us@,taylors.edu.my

\section{Su Dinh Thanh}

School of Public Finance, University of Economics Ho Chi Minh City,

Vietnam

Dinbthanb@ueh.edu.vn

\section{Felicia Hui Ling Chong}

School of Accounting and Finance, Taylor's University,

Malaysia

HuiLing.Chong@taylors.edu.my

Abstract. This study aims at investigating the influence of institutions and economic openness on credit cycles in a global sample. Six institutional quality indicators combined with net inward FDI and trade openness are collected to estimate, respectively, the effects of institutions and economic openness on credit cycles. Our panel data covers 60 economies, including 32 low- and middle-income economies (LMEs) and 28 high-income economies (HIEs), the data ranging between 2003 and 2017. Although better institutions tend to stimulate credit growth, they significantly stabilize credit cycles. These findings are documented with significant results in LMEs while it is less obvious in HIEs.

Received: January, 2020 1st Revision: August, 2020

Accepted: November, 2020

DOI:

$10.14254 / 2071$ $8330.2020 / 13-4 / 16$

Keywords: institutional quality, FDI, trade openness, credit cycles.

JEL Classification: E02, E51, F13, F21, H81 


\section{INTRODUCTION}

After the 2008 global financial crisis, we observed a deep economic recession in both advanced and lower income economies. Among many other things, this crisis and recession have been highlighting the importance of credit cycles. According to empirical studies (Ramos-Tallada, 2015, Phuc Nguyen et al., 2018), credit cycles have a strong impact on business cycles and macroeconomic stability through their effect on the aggregate demand (consumption and investment). Credit market cycles often lead to an increase in the macroeconomic disequilibrium such as higher inflation or financial instability. In a globalized context characterized by stronger financial integration, credit and macroeconomic cycles also lead to a contagion of turbulence between economies (Eickmeier \& Ng, 2015).

Many empirical studies identify the links between the credit cycles and major economic factors such as economic growth, monetary policy, fiscal policy, inflation, interest rate, business cycles, banking system conditions (Matsuyama et al., 2016, Vo and Nguyen, 2014). It is also worth mentioning the existence of studies investigating the relationship between credit cycles and such external factors as inflow capital and trade (see Magud \& Vesperoni (2015)). The aspect of credit cycles is usually addressed from two different perspectives: (i) economic models for estimating the equilibrium of credit in a given economy (see Kiss et al. (2006)); and (ii) estimating the relationship between the standard deviation of credit and a set of economic, demographic or financial factors (Rubaszek \& Serwa, 2014). The majority of the existing studies focused on the determinants of overall credit level without considering the effects of the determinants in the periods of booming and recession at the credit market (see, e.g., Shen et al. (2016)). Furthermore, the existing literature dealing with this issue doesn't really consider the role of institutions in a comprehensive perspective with the influence of the inward FDI and trade openness in the dynamics of credit market cycles.

This study provides empirical evidence on how credits are affected by institutions and economic openness. Institutions can be defined as "the rule of game" in a society as they are defining market rules and constraints on human and economic agencies' behaviors. From such a perspective, good institutional quality is said to reduce the asymmetric information problem and risks by increasing market efficiency, asset allocation efficiency and property rights (Acemoglu \& Robinson, 2008, Canh et al., 2018a, Canh et al., 2018b). Such an influential role might have a significant impact on the credit market. This article aims at investigating this aspect.

Our study is done in three steps. First, our argument is to estimate the influence of institutions on credit cycles by interpreting their impacts on economic incentives. Second, we estimate the equilibrium for credit by using four different indicators of credit market on a panel data of 60 economies in the period from 2003 to 2017. Afterwards, we calculate the residuals of our estimation and divide them by the fitted value from estimations to measure the credit cycles. Credit cycles are used as a dependent variable in our main model to which we add institutional indicators, inward FDI, and trade openness to describe the dynamics of these credit cycles. Moreover, we define a dummy variable for the credit cycles: the dummy variable is 0 if the credit cycle value is negative (to proxy a recession in credit market) and it is 1 if the credit cycle value is positive (to proxy a boom in credit market). The logit model is applied to estimate the influences of institutions and economic openness on the probability of transiting from a recession to a boom period in credit market. Finally, we estimate these institutional influences combined with the one of economic openness on the credit cycle values to investigate the roles of institutions and economic openness in stabilizing the credit cycles. We use four kinds of credit categories including the ratio of private credit by deposit money banks to GDP (\%), the ratio of private credit by deposit money banks and other financial institutions to GDP (\%), the ratio of domestic credit to private sector by banks to GDP (\%), and the ratio of domestic credit to private sector to GDO $(\%)$ in order to explore the potential effects of institutions, 
inward FDI, and trade openness on different cycles in credit market. We also use these four indicators to check for robustness. This article contributes to the literature dealing with credit cycles by defining the influences of institutions and economic openness on credit cycles.

The rest of this study is organized as follows. Section 2 outlines the literature review on the determinants of credit equilibrium and credit cycles. Section 3 describes our methodology. Section 4 discusses our results while Section 5 concludes this research with some policy suggestions.

\section{LITERATURE REVIEW}

This section first presents an overview of the works dealing with the major determinants of credit cycles including institutional indicators, inward FDI and trade openness.

The existing literature mainly confirmed that the GDP growth is an important driver for the credit demand (Backé \& Wójcik, 2008). Chen et al. (2012) used the real GDP as the first explanatory variable in credit cycle in the US while Duprey (2012), Karfakis (2013) or Dees (2016) used the real GDP as the major explanatory variable explaining banks' behavior in the context of macroeconomic fluctuations.

Broadly speaking, credit cycles are defined as an absolute and relative deviation from the credit equilibrium (Aikman et al., 2015). Several determinants influencing credit cycles can be mentioned. First, the economic growth that is mainly associated with the demand side of credit channels (Aysun \& Hepp, 2013). Economic growth is expected to improve financial conditions of firms and households contributing therefore to a high level of credit (Kiss et al., 2006, Backé \& Wójcik, 2008). However, an increase in the economic growth may lead to an excessive demand for credit leading to a potential demand credit booms (Lambertini et al., 2013). Second, the capital and risk conditions of the financial systems are also important determinants for the credit supply following bank lending channel literature (Altunbas et al., 2010, Canh, 2016)

FDIs are expected to contribute to additional capital into the host country's aggregate investment, however, it can trigger a lending boom in credit market (Mendoza and Terrones, 2008). A high level of inward capital flows also simulate the development of the financial sector while the domestic banking system can transform illiquid assets into liquid assets accessible although nationals may over-borrow abroad and over-lend domestically (Krugman, 1999). Even though inward FDI flows usually have a positive impact on the economic growth through a spillover effect (due to productivity, technological transfer and human capital), it is not necessary true in all cases (Huynh et al., 2020, Nguyen et al., 2018b, Nguyen et al. 2018c). Samarina and Bezemer (2016) showed that domestic banking system in emerging economies does not have the ability to transform assets due to the lack of financial tools - Such situation significantly reduces the effect of inward FDI on credit volatility. In the same vein, Igan and Tan (2015) emphasized that only nonFDI capital inflows boost credit growth to household and corporate sectors. Although, macroeconomic factors have a impact on the credit cycles, these effects are different across the countries (Dees, 2016). Differences in regulation of the financial system usually explain the differences in credit cycles (Jiménez et al., 2005, Imran \& Nishat, 2013).

In the literature dealing with institutions, several studies investigated the relationship between the latter and the economic growth (Nguyen et al., 2018a, Phuc Nguyen et al., 2018, Thong \& Canh, 2016). Other studies focused on the link between institutional quality and several macroeconomic factors such as trade, firm growth, productivity, market efficiency and competitiveness of firms (Araujo et al., 2016, Canh et al., 2018b; Luo \& Schinckus 2015). Generally speaking, institutions contribute to an improvement of the social, political and economic structures influencing therefore the development of credit market. Precisely, a higher institutional quality might simulate banks and other financial institutions in lending and expanding their credit portfolios. Indeed, a good institutional quality improves the regulations and it increases trust between 
banks and their customers leading to the reduction of asymmetric information and risks. This effect has a positive impact on the lending of banks and other financial institutions. In such case, if credit level is under equilibrium, an improvement in the institutional quality would increase it to equilibrium level or even higher exacerbating potentially the credit far away from the equilibrium. Institutions also impact the credit volatility through their influences on the changes in credit demand. A good institutional quality actually simulates the entrepreneurship, innovation and competition (Herrera-Echeverri et al., 2014, Canh et al., 2018a).

It is worth mentioning that better institutions could help to curd the credit cycles since they increase the responsibility from both banks and economic agents. Herrera-Echeverri et al. (2014), for instance, found a strong positive relationship between the institutional quality and business generation while a high institutional quality may also influence the capital structures of companies: by reducing asymmetric information risk, firms are more keen to fund their activities by using more equity (Bucsa et al. 2011; Petacchi, 2015). Such observations contribute to the stabilization of the credit cycles. The following section presents our data and the way we do analyze them.

\section{METHODOLOGY}

In this section, we present in detail our methodology. We use a traditional method (Kiss et al. (2006) or Igan and Tan (2015)) to estimate the equilibrium of credit market by integrating its lag influence and time effects; precisely the level of credit can be summarized as,

$$
\text { Credit }_{\text {it }}=\beta_{0}+\beta_{1} \text { Credit }_{i t-1}+\beta_{2} T_{t}+\varepsilon_{i t}
$$

where, $i$ is the country and $t$ stands for time (year); Credit is the credit level that is captured through 4 indicators: ratio of private credit by deposit money banks to GDP (Credit1), the ratio of private credit by deposit money banks and other financial institutions to GDP (Credit2), the ratio of domestic credit to private sector by banks to GDP (Credit3), the ratio of domestic credit to private sector to GDP (Credit4). We use different proxies to capture the level of credit as robustness check, to be sure that our analysis of the credit provides acceptable results. Afterwards, the proxy of the credit level is regressed in logarithm form to reduce the heteroscedasticity. $\mathrm{T}$ is time variable, which is the year; $\beta$ is a coefficient; $\varepsilon \varepsilon$ is the classical residuals. After having estimated the equilibrium of credit level, we focus on the residuals and we divide them by the fitted value of each equation to measure credit cycles. In line with Igan and Tan (2015), we then model dynamics equation to test the effects of institutions, inward FDI, and trade openness on the credit volatility; more precisely,

$$
\begin{gathered}
\text { Crevo }_{i t}=\alpha_{0}+\alpha_{\mathrm{j}} \mathrm{X}_{\mathrm{it}}+\gamma_{1} \text { Trade }_{i t}+\gamma_{2} \mathrm{FDI}_{\mathrm{it}}+\gamma_{3} \mathrm{INST}_{\mathrm{it}}+\varepsilon_{\mathrm{it}} \\
\text { Creditcycle }_{i t}=\text { Creditcycle }_{i t-1}+\delta_{i} X_{i t}+\alpha_{1} \text { Fdilingdp }_{i t}+\alpha_{2} \text { Tradegdp }_{i t}+\alpha_{3} \text { Inqua }_{i t}+u_{i t}
\end{gathered}
$$

where, Crevo denotes for four different proxies of credit cycles, respectively; FDI is the ratio of net inward FDI to GDP; Trade is the ratio of total trade value to GDP; INST refers to the institutional quality, which is presented by the average of the six World Bank institutional indicators including government effectiveness (Goveff), regulatory quality (Requa), rule of law (Law), and control of corruption (Concor), political stability (Politic), and voice and accountability (Voice), respectively. These World Governance Indicators are scaled from -2.5 to +2.5 for each indicator implying that the higher value means better institutional quality. $\mathrm{X}$ is a set of control variables including the bank capital to total assets (Cap), the bank return on assets (ROA), the bank risk proxying by the bank Z-score index (Risk), real GDP growth rate (GDPg). 
In this study, we collect data from different sources including World Development Indicators (WDI), World Governance Indicators (WGI), and Global Financial Development Database (GFDD) (World Bank). Due to the availability from World Governance Indicators and from Global Financial Development Database1, our final sample has 60 economies over the period 2003-2017. The sample is divided into two subsamples including 32 Low and Middle income economies (LMEs) and 28 High income economies (HIEs) to examine the combined effect of institutions and economic integration on the credit cycles for different income levels.

The description of four our proxies for credit levels are reported in Table 1A hereafter.

Table 1A

Data description of credit levels

\begin{tabular}{|c|c|c|c|c|c|c|c|}
\hline Variable & Definitions & Source & Obs & Mean & Std. Dev. & Min & Max \\
\hline \multicolumn{8}{|c|}{ Full sample } \\
\hline Credit1 & $\begin{array}{l}\text { Private credit by deposit money banks } \\
\text { to GDP }(\%)\end{array}$ & GFDD & 780 & 62.383 & 43.472 & 5.590 & 219.117 \\
\hline Credit2 & $\begin{array}{l}\text { Private credit by deposit money banks } \\
\text { and other financial institutions to } \\
\text { GDP }(\%)\end{array}$ & GFDD & 780 & 67.348 & 48.069 & 5.590 & 219.117 \\
\hline Credit3 & $\begin{array}{l}\text { Domestic credit to private sector by } \\
\text { banks }(\% \text { of GDP) }\end{array}$ & WDI & 779 & 64.033 & 43.341 & 5.637 & 233.211 \\
\hline Credit4 & $\begin{array}{l}\text { Domestic credit to private sector }(\% \\
\text { of GDP) }\end{array}$ & WDI & 779 & 68.743 & 47.879 & 5.682 & 233.211 \\
\hline GDP & GDP (current US\$) & WDI & 780 & $8.7 \mathrm{E}+11$ & $2.2 \mathrm{E}+12$ & $1.9 \mathrm{E}+09$ & $1.8 \mathrm{E}+13$ \\
\hline \multicolumn{8}{|c|}{32 Low and Middle income economies (LMEs) } \\
\hline Credit1 & $\begin{array}{l}\text { Private credit by deposit money banks } \\
\text { to GDP }(\%)\end{array}$ & GFDD & 416 & 38.337 & 27.340 & 5.590 & 140.400 \\
\hline Credit2 & $\begin{array}{l}\text { Private credit by deposit money banks } \\
\text { and other financial institutions to } \\
\text { GDP }(\%)\end{array}$ & GFDD & 416 & 39.798 & 28.795 & 5.590 & 147.086 \\
\hline Credit3 & $\begin{array}{l}\text { Domestic credit to private sector by } \\
\text { banks (\% of GDP) }\end{array}$ & WDI & 416 & 40.653 & 27.995 & 5.637 & 152.541 \\
\hline Credit4 & $\begin{array}{l}\text { Domestic credit to private sector }(\% \\
\text { of GDP) }\end{array}$ & WDI & 416 & 42.127 & 29.317 & 5.682 & 152.552 \\
\hline GDP & GDP (current US\$) & WDI & 416 & $4.5 \mathrm{E}+11$ & $1.2 \mathrm{E}+12$ & $1.9 \mathrm{E}+09$ & $1.11 \mathrm{E}+13$ \\
\hline \multicolumn{8}{|c|}{28 High income economies (HIEs) } \\
\hline Credit1 & $\begin{array}{l}\text { Private credit by deposit money banks } \\
\text { to GDP }(\%)\end{array}$ & GFDD & 364 & 89.864 & 42.203 & 20.249 & 219.117 \\
\hline Credit2 & $\begin{array}{l}\text { Private credit by deposit money banks } \\
\text { and other financial institutions to } \\
\text { GDP }(\%)\end{array}$ & GFDD & 364 & 98.833 & 46.334 & 20.250 & 219.117 \\
\hline Credit3 & $\begin{array}{l}\text { Domestic credit to private sector by } \\
\text { banks (\% of GDP) }\end{array}$ & WDI & 363 & 90.828 & 42.315 & 13.350 & 233.211 \\
\hline Credit4 & $\begin{array}{l}\text { Domestic credit to private sector }(\% \\
\text { of GDP) }\end{array}$ & WDI & 363 & 99.246 & 46.844 & 13.353 & 233.211 \\
\hline GDP & GDP (current US\$) & WDI & 364 & $\begin{array}{r}1.3 \mathrm{E}+1 \\
2\end{array}$ & $\begin{array}{r}2.9 \mathrm{E}+1 \\
2\end{array}$ & $\begin{array}{r}9.8 \mathrm{E}+0 \\
9\end{array}$ & $\begin{array}{r}1.8 \mathrm{E}+1 \\
3\end{array}$ \\
\hline
\end{tabular}

1 The data from WGIs is available from 2002, while the data from GFDD is available until 2017 (see https://www.worldbank.org/en/publication/gfdr/data/global-financial-development-database). Moreover, most of variables have data from 2003. Therefore, the period 2003-2017 is best sample for empirical investigation. 
This article then uses the robust pool OLS to estimate equation [1] (all results can be provided on request). The description of final variables is presented in Table $1 \mathrm{~B}$.

Table 1B

Data calculations and descriptions

\begin{tabular}{|c|c|c|c|c|c|c|c|}
\hline Variable & Calculations & $\begin{array}{l}\text { Sourc } \\
\mathrm{e}\end{array}$ & Obs & Mean & $\begin{array}{l}\text { Std. } \\
\text { Dev. }\end{array}$ & Min & Max \\
\hline Cre1vo & $\begin{array}{l}\text { The percentage of residual from estimation in equation [1] to its } \\
\text { fitted value for } \boldsymbol{C} \text { redit } 1\end{array}$ & & 720 & 0.0002 & 0.441 & -2.297 & 1.668 \\
\hline Cre2vo & $\begin{array}{l}\text { The percentage of residual from estimation in equation [1] to its } \\
\text { fitted value for } \boldsymbol{C} \text { redit } \boldsymbol{2}\end{array}$ & & 720 & 0.0002 & 0.442 & -2.303 & 1.669 \\
\hline Cre3vo & $\begin{array}{l}\text { The percentage of residual from estimation in equation [1] to its } \\
\text { fitted value for } \boldsymbol{C} \text { redit } 3\end{array}$ & & 719 & 0.0002 & 0.478 & -2.605 & 2.252 \\
\hline Cre4vo & $\begin{array}{l}\text { The percentage of residual from estimation in equation [1] to its } \\
\text { fitted value for } \boldsymbol{C r} \text { redit } 4\end{array}$ & & 719 & 0.0002 & 0.476 & -2.605 & 2.234 \\
\hline Cap & Bank capital to total assets $(\%)$ & $\begin{array}{l}\text { GFD } \\
\mathrm{D}\end{array}$ & 780 & 9.405 & 3.776 & 2.700 & 26.50 \\
\hline $\mathrm{ROA}$ & Bank return on assets $(\%$, after tax $)$ & $\begin{array}{l}\text { GFD } \\
\text { D }\end{array}$ & 780 & 1.163 & 1.153 & -8.522 & 8.316 \\
\hline Risk & Bank Z-score & $\begin{array}{l}\text { GFD } \\
\text { D }\end{array}$ & 780 & 13.442 & 8.598 & 0.017 & 53.51 \\
\hline GDPg & Real GDP growth (annual \%) & WDI & 780 & 3.720 & 3.689 & -14.72 & 17.32 \\
\hline Trade & Trade $(\%$ of GDP $)$ & WDI & 780 & 93.10 & 71.15 & 21.58 & 442.62 \\
\hline FDI & Foreign direct investment, net inflows ( $\%$ of GDP) & WDI & 780 & 5.881 & 12.58 & -58.32 & 252.31 \\
\hline INST & Average of six institutional indicators & & 780 & 0.357 & 0.837 & -1.178 & 1.970 \\
\hline Concor & Control of Corruption (Estimate value) & WGI & 780 & 0.375 & 1.036 & -1.394 & 2.470 \\
\hline Goveff & Government Effectiveness (Estimate value) & WGI & 780 & 0.490 & 0.911 & -0.997 & 2.437 \\
\hline Politic & $\begin{array}{l}\text { Political Stability and Absence of Violence/Terrorism (Estimate } \\
\text { value) }\end{array}$ & WGI & 780 & 0.041 & 0.875 & -2.810 & 1.688 \\
\hline Requa & Regulatory Quality (Estimate value) & WGI & 780 & 0.542 & 0.813 & -1.296 & 2.261 \\
\hline Law & Rule of Law (Estimate value) & WGI & 780 & 0.370 & 0.960 & -1.251 & 2.100 \\
\hline Voice & Voice and Accountability (Estimate value) & WGI & 780 & 0.324 & 0.871 & -1.907 & 1.801 \\
\hline
\end{tabular}

Note: GFDD is Global Financial Development Database, WDI is World Development Indicators, WGI is

World Governance Indicators (World Bank)

Methodologically speaking, there is a potential problem of endogeneity. Since some studies suggested that credit cycles might influence economic stability \indicators. To handle with this problem, we use the Granger-causality test of Dumitrescu and Hurlin (2012) to examine the causality between independent variables and dependent variable. The results are presented in the Table 2.

Table 2

Correlation matrix

\begin{tabular}{|c|c|c|c|c|}
\hline Correlation & Cre1vo & Cre2vo & Cre3vo & Cre4vo \\
\hline Cre2vo & $0.991 * * *$ & 1.000 & & \\
\hline $\mathrm{p}$-value & 0.000 & & & \\
\hline Cre3vo & $0.867 * * *$ & $0.861 * * *$ & 1.000 & \\
\hline $\mathrm{p}$-value & 0.000 & 0.000 & & \\
\hline Cre4vo & $0.866 * * *$ & $0.867 * * *$ & $0.991 * * *$ & 1.000 \\
\hline $\mathrm{p}$-value & 0.000 & 0.000 & 0.000 & \\
\hline Cap & $0.113 * * *$ & $0.123 * * *$ & $0.094 * *$ & $0.103 * * *$ \\
\hline p-value & 0.002 & 0.001 & 0.012 & 0.006 \\
\hline ROA & $0.251 * * *$ & $0.253 * * *$ & $0.273 * * *$ & $0.275^{* * *}$ \\
\hline $\mathrm{p}$-value & 0.000 & 0.000 & 0.000 & 0.000 \\
\hline Risk & $-0.064 *$ & $-0.066^{*}$ & -0.053 & -0.054 \\
\hline $\mathrm{p}$-value & 0.089 & 0.078 & 0.159 & 0.147 \\
\hline GDPg & $0.384 * * *$ & $0.382^{* * *}$ & $0.452^{* * *}$ & $0.447 * * *$ \\
\hline p-value & 0.000 & 0.000 & 0.000 & 0.000 \\
\hline Trade & -0.048 & -0.056 & -0.040 & -0.043 \\
\hline$p$-value & 0.198 & 0.135 & 0.289 & 0.255 \\
\hline
\end{tabular}




\begin{tabular}{|c|c|c|c|c|c|c|c|c|c|c|}
\hline FDI & & \multicolumn{4}{|c|}{-0.001} & \multicolumn{2}{|c|}{-0.005} & \multicolumn{2}{|l|}{-0.003} & -0.005 \\
\hline & $\mathrm{p}$-value & \multicolumn{4}{|c|}{0.973} & \multicolumn{2}{|l|}{0.902} & \multicolumn{2}{|l|}{0.940} & 0.896 \\
\hline INST & & \multicolumn{4}{|c|}{$-0.118 * * *$} & \multicolumn{2}{|c|}{$-0.124 * * *$} & $-0.101 * * *$ & & $-0.106 * * *$ \\
\hline & $\mathrm{p}$-value & \multicolumn{4}{|c|}{0.002} & \multicolumn{2}{|c|}{0.001} & \multicolumn{2}{|l|}{0.007} & 0.004 \\
\hline Concor & & \multicolumn{4}{|c|}{$-0.105^{* * *}$} & -0.112 & $* * *$ & $-0.091 * *$ & -0.0 & \\
\hline & $p$-value & 0.00 & & & & 0.003 & & 0.015 & 0.01 & \\
\hline Goveff & & -0.0 & *** & & & -0.102 & $2 * * *$ & $-0.082 * *$ & -0.08 & \\
\hline & $p$-value & 0.01 & & & & 0.006 & & 0.028 & 0.02 & \\
\hline Politic & & -0.1 & **** & & & -0.112 & $* * *$ & $-0.086 * *$ & -0.0 & \\
\hline & p-value & 0.00 & & & & 0.003 & & 0.021 & 0.01 & \\
\hline Requa & & -0.0 & $* *$ & & & -0.094 & & $-0.079 * *$ & -0.08 & \\
\hline & $\mathrm{p}$-value & 0.01 & & & & 0.011 & & 0.035 & 0.02 & \\
\hline Law & & -0.1 & *** & & & -0.127 & $7 * *$ & $-0.101 * * *$ & -0.10 & \\
\hline & p-value & 0.00 & & & & 0.001 & & 0.007 & 0.00 & \\
\hline Voice & & -0.1 & $* * *$ & & & -0.132 & $2 * * *$ & $-0.115 * * *$ & -0.12 & \\
\hline & $p$-value & 0.00 & & & & 0.000 & & 0.002 & 0.00 & \\
\hline Correlation & & Cap & & & & & Risk & GDPg & Trade & FDI \\
\hline ROA & & 0.339 & & & & & & & & \\
\hline & $\mathrm{p}$-value & 0.000 & & & & & & & & \\
\hline Risk & & 0.037 & & & & & 1.000 & & & \\
\hline & $\mathrm{p}$-value & 0.297 & & & & & & & & \\
\hline GDPg & & 0.209 & & & $* * *$ & & $0.071 * *$ & 1.000 & & \\
\hline & p-value & 0.000 & & & & & 0.048 & & & \\
\hline Trade & & -0.07 & & & & & $0.219 * * *$ & 0.038 & 1.000 & \\
\hline & p-value & 0.044 & & & & & 0.000 & 0.288 & & \\
\hline FDI & & -0.04 & & & & & 0.108 & 0.026 & $0.473 * * *$ & 1.000 \\
\hline & p-value & 0.174 & & & & & 0.003 & 0.471 & 0.000 & \\
\hline INST & & -0.49 & & & $6 * * *$ & & $0.075 * *$ & $-0.328^{* * *}$ & $0.391 * * *$ & \begin{tabular}{|l}
$0.214^{* * *}$ \\
\end{tabular} \\
\hline & p-value & 0.000 & & & & & 0.037 & 0.000 & 0.000 & 0.000 \\
\hline Concor & & -0.46 & & & $4 * * *$ & & $0.071 * *$ & $-0.271 * * *$ & $0.392 * * *$ & $0.220 * * *$ \\
\hline & p-value & 0.000 & & & & & 0.048 & 0.000 & 0.000 & 0.000 \\
\hline Goveff & & -0.48 & & & $6 * * *$ & & $0.102 * * *$ & $-0.293 * * *$ & $0.412 * * *$ & $0.192 * * *$ \\
\hline & $\mathrm{p}$-value & 0.000 & & & & & 0.005 & 0.000 & 0.000 & \begin{tabular}{|l|}
0.000 \\
\end{tabular} \\
\hline Politic & & -0.34 & & & $6 * * *$ & & $0.069 *$ & $-0.256 * * *$ & $0.415 * * *$ & \begin{tabular}{|l}
$0.212 * * *$ \\
\end{tabular} \\
\hline & p-value & 0.000 & & & & & 0.054 & 0.000 & 0.000 & 0.000 \\
\hline Requa & & -0.42 & & & $6 * * *$ & & $0.115^{* * *}$ & $-0.319 * * *$ & $0.433^{* * *}$ & $0.228 * * *$ \\
\hline & p-value & 0.000 & & & & & 0.001 & 0.000 & 0.000 & 0.000 \\
\hline Law & & -0.49 & & & $6 * * *$ & & $0.074 * *$ & $-0.310 * * *$ & $0.377 * * *$ & $0.192 * * *$ \\
\hline & p-value & 0.000 & & & & & 0.038 & 0.000 & 0.000 & 0.000 \\
\hline Voice & & -0.49 & & & $9 * * *$ & & -0.018 & $-0.366 * * *$ & $0.122 * * *$ & $0.135^{* * *}$ \\
\hline & p-value & 0.000 & & & & & 0.621 & 0.000 & 0.001 & 0.000 \\
\hline Correlation & INST & & $\mathrm{Co}$ & & Go & jeff & Politic & Requa & Law & Voice \\
\hline Concor & $0.967^{*}$ & & 1.0 & & & & & & & \\
\hline $\mathrm{p}$-value & 0.000 & & & & & & & & & \\
\hline Goveff & $0.956^{*}$ & & 0.9 & & 1.00 & & & & & \\
\hline $\mathrm{p}$-value & 0.000 & & 0.0 & & & & & & & \\
\hline Politic & $0.822^{*}$ & & 0.7 & & 0.6 & 6*** & 1.000 & & & \\
\hline$p$-value & 0.000 & & 0.0 & & 0.0 & & & & & \\
\hline Requa & $0.958^{*}$ & & 0.9 & & 0.9 & $2 * * *$ & $0.729 * * *$ & 1.000 & & \\
\hline $\mathrm{p}$-value & 0.000 & & 0.0 & & 0.00 & & 0.000 & & & \\
\hline Law & $0.975^{*}$ & & 0.9 & & 0.9 & $5 \% * *$ & $0.750 * * *$ & $0.945 * * *$ & 1.000 & \\
\hline $\mathrm{p}$-value & 0.000 & & 0.0 & & 0.00 & & 0.000 & 0.000 & & \\
\hline Voice & $0.823^{*}$ & & 0.7 & & 0.7 & $3 * * *$ & $0.616 * * *$ & $0.740 * * *$ & $0.746 * * *$ & 1.000 \\
\hline $\mathrm{p}$-value & 0.000 & & 0.0 & & 0.0 & & 0.000 & 0.000 & 0.000 & \\
\hline
\end{tabular}

Note: ${ }^{*},{ }^{* *},{ }^{* * *}$ are significant levels at $10 \%, 5 \%, 1 \%$, respectively.

Granger-causality tests show that there is a mutual causality between the independent variables and dependent variables. To solve this issue, we estimate the Equation [2] by using the lags of all independent variables: 


$$
\begin{gathered}
\text { Crevo }_{\mathrm{it}}=\alpha_{0}+\alpha_{\mathrm{j}} \mathrm{X}_{\mathrm{it}-1}+\gamma_{1} \text { Trade }_{\mathrm{it}-1}+\gamma_{2} \mathrm{FDI}_{\mathrm{it}-1}+\gamma_{3} \mathrm{INST}_{\mathrm{it}-1}+\varepsilon_{\mathrm{it}} \\
\text { Creditcycle }_{i t}=\text { Creditcycle }_{i t-1}+\delta_{i} X_{i t}+\alpha_{1} \text { Fdilingdp }_{i t}+\alpha_{\mathbf{2}} \text { Tradegd }_{i t}+\alpha_{\mathbf{3}} \text { Inqua }_{i t}+u_{i t}
\end{gathered}
$$

In the estimation this equation, we use the robust pool OLS to tackle the problem of heteroscedasticity of the sample. In addition, we use a dummy variable as explained hereafter,

$$
\{\mathrm{DUM}=0 \text { if Crevo }<0 \text { DUM }=1 \text { if Crevo } \geq 0
$$

This dummy variable (DUM) is used to examine the effect of institutions and the economic integration on the possibility of a credit boom in the financial system. More specifically,

$$
\operatorname{DUM}_{\mathrm{it}}=\alpha_{0}+\alpha_{\mathrm{j}} \mathrm{X}_{\mathrm{it}-1}+\gamma_{1} \text { Trade }_{\mathrm{it}-1}+\gamma_{2} \mathrm{FDI}_{\mathrm{it}-1}+\gamma_{3} \mathrm{INST}_{\mathrm{it}-1}+\varepsilon_{\mathrm{it}}
$$

By estimating the equation [5], a logit model is used since the dependent variable is a dummy variable. The estimation of the equation [3] can take two forms: positive credit cycle (Crevo $>=0$ ) and negative credit cycles $($ Crevo $<0)$. The following section aims at investigating these potential boom and recession on the credit market.

\section{EMPIRICAL RESULTS AND DISCUSSION}

\subsection{The Global Sample}

The effect of the institutional quality, inward FDI, and trade openness on the private sector credit cycles in private credit by deposit money banks to GDP (Cre1vo) are presented in the table 3 hereafter.

First, we examine the effect of control variables on the credit cycles for private credit by deposit money banks. The positive coefficient for bank capital (Cap) and bank profitability (ROA) mean that the banking system with highly capitalized profitable conditions have all conditions to increase their credit growth. The results are in line with previous literature according to which highly capitalized profitable banking system have higher capability to supply credit (Altunbaş et al., 2002). Meanwhile, banking systems with a higher risk profile (Risk) has a lower credit growth rate, which is expected since riskier banking systems have to reduce their credit supply (Bernanke and Gertler, 1995). Such observation is however in opposition with some empirical findings claiming that riskier banking systems have to implement several risk-taking activities (de Moraes et al., 2016, Dell'Ariccia et al., 2014). The positive coefficient for logarithm of GDP per capita is at $1 \%$ significance, suggesting that the economic growth leads to an increase of the credit level. In other words, a high economic growth favors credit cycles in line with existing studies on the issues (Kiss et al. (2006), Igan and Tan (2015), Mendoza and Terrones (2008), Chen et al. (2012), Duprey (2012), Apostoaie and Percic, 2014)). 
Granger-causality test

\begin{tabular}{|c|c|c|c|c|c|c|c|c|}
\hline \multicolumn{9}{|c|}{ Full sample } \\
\hline \multirow[t]{2}{*}{$\mathbf{X}$ Variable } & \multicolumn{2}{|c|}{$\begin{array}{c}\mathbf{X} \text { does not Granger- } \\
\text { cause Cre1vo }\end{array}$} & \multicolumn{2}{|c|}{$\begin{array}{c}\text { X does not Granger- } \\
\text { cause } \text { Cre2vo }\end{array}$} & \multicolumn{2}{|c|}{$\begin{array}{c}\mathbf{X} \text { does not Granger- } \\
\text { cause Cre3vo }\end{array}$} & \multicolumn{2}{|c|}{$\begin{array}{c}\mathbf{X} \text { does not Granger- } \\
\text { cause Cre4vo }\end{array}$} \\
\hline & Z-bar & p-value & Z-bar & $\mathrm{p}$-value & Z-bar & p-value & Z-bar & p-value \\
\hline Cap & $11.93^{* * *}$ & 0.000 & $12.45^{* * *}$ & 0.000 & $9.743^{* * *}$ & 0.000 & $9.529 * * *$ & 0.000 \\
\hline ROA & $8.041 * * *$ & 0.000 & $7.487 * * *$ & 0.000 & $7.947 * * *$ & 0.000 & $9.314^{* * *}$ & 0.000 \\
\hline Risk & $14.81 * * *$ & 0.000 & $10.08^{* * *}$ & 0.000 & $12.58^{* * *}$ & 0.000 & $10.35^{* * *}$ & 0.000 \\
\hline GDPg & $7.778^{* * *}$ & 0.000 & $6.193^{* * *}$ & 0.000 & $6.289 * * *$ & 0.000 & $5.529 * * *$ & 0.000 \\
\hline Trade & 0.233 & 0.815 & 0.261 & 0.794 & $2.742^{* * *}$ & 0.006 & $2.158^{* *}$ & 0.031 \\
\hline FDI & $7.534 * * *$ & 0.000 & $6.864 * * *$ & 0.000 & $6.449 * * *$ & 0.000 & $6.298^{* * *}$ & 0.000 \\
\hline INST & $3.699 * * *$ & 0.000 & $3.683^{* * *}$ & 0.000 & $4.296^{* * *}$ & 0.000 & $3.666^{* * *}$ & 0.000 \\
\hline Concor & 0.479 & 0.631 & 0.180 & 0.857 & 0.205 & 0.837 & 0.356 & 0.721 \\
\hline Goveff & $4.429 * * *$ & 0.000 & $4.914^{* * *}$ & 0.000 & $3.053^{* * *}$ & 0.002 & $3.014^{* * *}$ & 0.003 \\
\hline Politic & $3.171 * * *$ & 0.002 & $3.878^{* * *}$ & 0.000 & $2.362^{* *}$ & 0.018 & $4.323 * * *$ & 0.000 \\
\hline Requa & $2.470 * *$ & 0.013 & $3.176^{* * *}$ & 0.002 & $2.771 * * *$ & 0.006 & $4.289 * * *$ & 0.000 \\
\hline Law & $4.735^{* * *}$ & 0.000 & $5.491 * * *$ & 0.000 & $3.311 * * *$ & 0.001 & $3.325^{* * *}$ & 0.001 \\
\hline Voice & 1.284 & 0.199 & 1.489 & 0.136 & -0.647 & 0.517 & 0.005 & 0.996 \\
\hline \multirow[t]{2}{*}{$\mathbf{X}$ Variable } & \multicolumn{2}{|c|}{$\begin{array}{l}\text { Cre1vo does not } \\
\text { Granger-cause } \mathbf{X}\end{array}$} & \multicolumn{2}{|c|}{$\begin{array}{l}\text { Cre2vo does not } \\
\text { Granger-cause } \mathbf{X}\end{array}$} & \multicolumn{2}{|c|}{$\begin{array}{l}\text { Cre3vo does not } \\
\text { Granger-cause } \mathbf{X}\end{array}$} & \multicolumn{2}{|c|}{$\begin{array}{l}\text { Cre4vo does not } \\
\text { Granger-cause } \mathbf{X}\end{array}$} \\
\hline & Z-bar & $\mathrm{p}$-value & Z-bar & $\mathrm{p}$-value & Z-bar & $\mathrm{p}$-value & Z-bar & $\mathrm{p}$-value \\
\hline Cap & 1.495 & 0.134 & 1.884* & 0.059 & $4.042^{* * *}$ & 0.000 & $3.780^{* * *}$ & 0.000 \\
\hline $\mathrm{ROA}$ & $7.427 * * *$ & 0.000 & $7.296^{* * *}$ & 0.000 & $7.499 * * *$ & 0.000 & $7.711^{* * *}$ & 0.000 \\
\hline Risk & $4.792^{* * *}$ & 0.000 & $4.334^{* * *}$ & 0.000 & $4.710^{* * *}$ & 0.000 & $4.838^{* * *}$ & 0.000 \\
\hline GDPg & $16.34^{* * *}$ & 0.000 & $14.82^{* * *}$ & 0.000 & $8.470 * * *$ & 0.000 & $9.210^{* * *}$ & 0.000 \\
\hline Trade & $15.93^{* * *}$ & 0.000 & $14.90^{* * *}$ & 0.000 & $6.533^{* * *}$ & 0.000 & $6.513^{* * *}$ & 0.000 \\
\hline FDI & $5.107 * * *$ & 0.000 & $4.640^{* * *}$ & 0.000 & $2.802^{* * *}$ & 0.005 & $2.432^{* *}$ & 0.015 \\
\hline INST & 0.014 & 0.988 & 0.156 & 0.875 & -0.941 & 0.346 & -1.348 & 0.177 \\
\hline Concor & 0.071 & 0.943 & 1.091 & 0.275 & -0.363 & 0.716 & -0.285 & 0.775 \\
\hline Goveff & -1.077 & 0.281 & 0.228 & 0.819 & 0.049 & 0.960 & 0.084 & 0.932 \\
\hline Politic & $4.864 * * *$ & 0.000 & $3.752^{* * *}$ & 0.000 & $3.997 * * *$ & 0.000 & $4.150^{* * *}$ & 0.000 \\
\hline Requa & $2.052^{* *}$ & 0.040 & $1.951^{*}$ & 0.051 & $1.658^{*}$ & 0.097 & 1.398 & 0.162 \\
\hline Law & -0.373 & 0.708 & -0.377 & 0.705 & -0.923 & 0.355 & -1.108 & 0.267 \\
\hline Voice & $2.899 * * *$ & 0.004 & $2.334^{* *}$ & 0.019 & $3.298^{* * *}$ & 0.001 & $2.421 * *$ & 0.016 \\
\hline
\end{tabular}

Note: The Granger non-causality test of Dumitrescu \& Hurlin (2012) is used, H0: X does not Granger-cause $\mathrm{Y}, \mathrm{H1}$ : X does Granger-cause Y for at least one panelvar (country). *, **, *** is significant levels at $10 \%$, $5 \%$, and $1 \%$, respectively.

The estimation of our explanatory variables is interesting: first, inward FDI has a significant positive effect on the private sector credit volatility (in line with existing empirical studies on the topic, (Mendoza \& Terrones, 2008) (Krugman, 1999). Our results also suggest that FDI has a significant contribution to the credit cycles. Trade openness exhibits a significant negative effect on the private sector credit volatility which also differs from some existing studies (Kaminsky and Reinhart, 1999). Precisely, our observations support the idea that trade openness can promote the development of the financial system and improve the quality of financial products (particularly credit contracts). In such context, trade openness increases the international competitiveness which stimulates productivity and economies of scale leading therefore to a rapid economic growth. This situation emphasizes the need for effective policies related to domestic capital and credit. The private sector credit volatility is also strongly affected by various institutional indicators. We find that the government effectiveness and the rule of law have a significant positive effect on the private sector credit volatility while the regulatory quality and the control of corruption have a significant negative effect. These results suggest that an improvement in government effectiveness promote the development 
of financial markets. Indeed, the government effectiveness creates incentives for the private sector to develop further economic and business expansion (increasing therefore the credit demand). As a result, banks (and other financial institutions) are keen to increase the credit portfolio they allocate to the private sector. The significant positive effect of the rule of law indicates that an improvement in this indicator contributes to the development of a more stable and transparent business environment favoring business activities (increasing therefore the credit demand).

Table 4

Institutions, Economic Openness and Credit volatility

\begin{tabular}{|c|c|c|c|c|c|c|c|}
\hline \multicolumn{8}{|c|}{ Part A: Robust Pool OLS regression } \\
\hline Dep. Var: Cre1vo & $(1)$ & $(2)$ & (3) & $(4)$ & $(5)$ & $(6)$ & $(7)$ \\
\hline Indep. Var: & INST & Concor & Goveff & Politic & Requa & Law & Voice \\
\hline \multirow[t]{2}{*}{ Cap(-1) } & 0.005 & 0.005 & 0.006 & 0.002 & 0.005 & 0.005 & 0.004 \\
\hline & {$[0.006]$} & {$[0.006]$} & {$[0.006]$} & {$[0.005]$} & {$[0.006]$} & {$[0.006]$} & {$[0.006]$} \\
\hline \multirow[t]{2}{*}{ ROA(-1) } & $0.084 * * *$ & $0.084 * * *$ & $0.086 * * *$ & $0.081 * * *$ & $0.084 * * *$ & $0.084 * * *$ & $0.083 * * *$ \\
\hline & {$[0.022]$} & {$[0.022]$} & {$[0.022]$} & {$[0.022]$} & {$[0.022]$} & {$[0.022]$} & {$[0.022]$} \\
\hline \multirow[t]{2}{*}{ Risk(-1) } & $-0.004 * *$ & $-0.004 * *$ & $-0.004 * *$ & $-0.004 * *$ & $-0.004 * *$ & $-0.004 * *$ & $-0.003 * *$ \\
\hline & {$[0.002]$} & {$[0.002]$} & {$[0.002]$} & {$[0.002]$} & {$[0.002]$} & {$[0.002]$} & {$[0.002]$} \\
\hline \multirow[t]{2}{*}{ GDPg(-1) } & $0.037 * * *$ & $0.036 * * *$ & $0.037 * * *$ & $0.035 * * *$ & $0.037 * * *$ & $0.036 * * *$ & $0.036 * * *$ \\
\hline & {$[0.006]$} & {$[0.005]$} & {$[0.005]$} & {$[0.005]$} & {$[0.006]$} & {$[0.006]$} & {$[0.006]$} \\
\hline \multirow[t]{2}{*}{ Trade(-1) } & $-0.001 * * *$ & $-0.001 * *$ & $-0.001 * * *$ & $-0.0005 * *$ & $-0.001 * * *$ & $-0.0006 * *$ & $-0.0004 *$ \\
\hline & {$[0.0002]$} & {$[0.0002]$} & {$[0.0002]$} & {$[0.0002]$} & {$[0.0002]$} & {$[0.0002]$} & {$[0.0002]$} \\
\hline \multirow[t]{2}{*}{ FDI(-1) } & $0.002 * *$ & $0.002 * *$ & $0.002 * *$ & $0.002 * *$ & $0.002 * *$ & $0.002 * *$ & $0.002 * *$ \\
\hline & {$[0.001]$} & {$[0.001]$} & {$[0.001]$} & {$[0.001]$} & {$[0.001]$} & {$[0.001]$} & {$[0.001]$} \\
\hline \multirow[t]{2}{*}{ INST(-1) } & $0.056 * *$ & $0.039 * *$ & $0.064 * * *$ & 0.020 & $0.066 * * *$ & $0.043 * *$ & $0.036^{*}$ \\
\hline & {$[0.024]$} & {$[0.018]$} & {$[0.022]$} & [0.019] & {$[0.026]$} & {$[0.021]$} & {$[0.022]$} \\
\hline \multirow[t]{2}{*}{ Cons. } & $-0.213^{* * *}$ & $-0.199 * * *$ & $-0.227 * * *$ & $-0.165^{* * *}$ & $-0.221 * * *$ & $-0.205^{* * *}$ & $-0.208^{* * *}$ \\
\hline & {$[0.061]$} & {$[0.058]$} & {$[0.062]$} & {$[0.050]$} & {$[0.062]$} & {$[0.061]$} & {$[0.065]$} \\
\hline $\mathrm{N}$ & 720 & 720 & 720 & 720 & 720 & 720 & 720 \\
\hline R-squared & 0.203 & 0.201 & 0.206 & 0.197 & 0.205 & 0.201 & 0.200 \\
\hline \multicolumn{8}{|c|}{ Part B: Logit regression } \\
\hline Dep. Var: DUM1 & $(1)$ & $(2)$ & (3) & $(4)$ & $(5)$ & (6) & $(7)$ \\
\hline \multirow{3}{*}{$\begin{array}{l}\text { Indep. Var: } \\
\text { Cap(-1) }\end{array}$} & INST & Concor & Goveff & Politic & Requa & Law & Voice \\
\hline & 0.012 & 0.014 & 0.022 & -0.006 & 0.009 & 0.014 & -0.007 \\
\hline & {$[0.026]$} & {$[0.026]$} & {$[0.027]$} & {$[0.025]$} & {$[0.026]$} & {$[0.027]$} & {$[0.025]$} \\
\hline \multirow[t]{2}{*}{ ROA(-1) } & $0.430 * * *$ & $0.439 * * *$ & $0.461 * * *$ & $0.394 * * *$ & $0.419 * * *$ & $0.434 * * *$ & $0.396 * * *$ \\
\hline & {$[0.121]$} & {$[0.123]$} & {$[0.127]$} & {$[0.116]$} & {$[0.120]$} & {$[0.123]$} & {$[0.116]$} \\
\hline \multirow{2}{*}{ Risk(-1) } & -0.011 & -0.011 & -0.013 & -0.011 & -0.012 & -0.012 & -0.011 \\
\hline & {$[0.009]$} & {$[0.009]$} & {$[0.009]$} & {$[0.009]$} & {$[0.009]$} & [0.009] & {$[0.009]$} \\
\hline \multirow[t]{2}{*}{ GDPg(-1) } & $0.145 * * *$ & $0.144 * * *$ & $0.148 * * *$ & $0.135 * * *$ & $0.147 * * *$ & $0.144 * * *$ & $0.133 * * *$ \\
\hline & {$[0.033]$} & {$[0.032]$} & {$[0.032]$} & {$[0.032]$} & {$[0.033]$} & {$[0.032]$} & {$[0.032]$} \\
\hline \multirow[t]{2}{*}{ Trade(-1) } & $-0.004 * *$ & $-0.004 * *$ & $-0.004 * * *$ & $-0.003 *$ & $-0.004 * *$ & $-0.004 * *$ & $-0.003 *$ \\
\hline & {$[0.002]$} & {$[0.002]$} & {$[0.002]$} & {$[0.002]$} & {$[0.002]$} & {$[0.002]$} & {$[0.001]$} \\
\hline \multirow[t]{2}{*}{ FDI(-1) } & $0.014 * *$ & $0.013 *$ & $0.015 * *$ & $0.014 *$ & $0.014 * *$ & $0.014 * *$ & $0.014 *$ \\
\hline & {$[0.007]$} & {$[0.007]$} & {$[0.007]$} & {$[0.007]$} & {$[0.007]$} & {$[0.007]$} & {$[0.008]$} \\
\hline \multirow[t]{2}{*}{ INST(-1) } & $0.275 * *$ & $0.257 * *$ & $0.364 * * *$ & 0.085 & $0.279 * *$ & $0.251 * *$ & 0.050 \\
\hline & {$[0.130]$} & [0.101] & {$[0.118]$} & {$[0.109]$} & {$[0.132]$} & [0.111] & {$[0.115]$} \\
\hline \multirow[t]{2}{*}{ Cons. } & $-0.854 * * *$ & $-0.862^{* * *}$ & $-0.999 * * *$ & $-0.583 * *$ & $-0.845^{* * *}$ & $-0.860 * * *$ & $-0.621 *$ \\
\hline & {$[0.309]$} & {$[0.300]$} & {$[0.311]$} & {$[0.271]$} & {$[0.309]$} & {$[0.307]$} & [0.319] \\
\hline $\mathrm{N}$ & 720 & 720 & 720 & 720 & 720 & 720 & 720 \\
\hline No. of countries & 60 & 60 & 60 & 60 & 60 & 60 & 60 \\
\hline
\end{tabular}

Note: Standard errors are in [. *,**, *** are significant levels at $10 \%, 5 \%, 1 \%$, respectively. 
The positive effect of institutions (INST) show that their improvement might cause an increase in risk explaining the raising of the private sector credit volatility. This is due to the positive effect of institutions on the economic incentives by promoting the economic activities. Moreover, the results from logit regression in Part B of table 4 show consistent findings meaning that our results can be reasonably considered as robust. Our results also mean that the credit cycles in better institutional environment are more likely moving from recession to booming periods. The Table 4 below documents the results for our estimations for two periods when the credit cycles are positive and negative, respectively.

The results show that all control variables were consistent in the period of positive cycles (Cre1vo >= $0)$. Interestingly, the institutions have a negative effect on the credit cycles. Moreover, in the period of negative cycles $(\mathrm{Cre} 1 \mathrm{vo}<0)$, the institutions have a positive effect meaning that a better institutional quality has lower influence on the credit cycles since it reduces the credit cycles in booming period while increases the credit cycles in recession period.

Meanwhile, both, the trade openness and FDI inflows have an opposite effect, they increase the credit cycles in booming period while they reduce credit cycle more deeply in recession period. This observation implies that the economic openness has a negative side by inducing higher probability of volatility in credit markets. This finding might explains the cause the financial crisis, which is consistent with many previous results (Igan \& Tan, 2015).

For robustness purpose, the economic analysis has been repeated with three different proxies of credit levels (the ratio of private credit by deposit money banks and other financial institutions to GDP (Credit2), the ratio of domestic credit to private sector by banks to GDP (Credit3), the ratio of domestic credit to private sector to GDP (Credit4)) respectively. All these proxies offer the same consistent results than our major analysis with Credit1. The detailed results of our robustness checks can be provided on request.

\subsection{The effects of institutions, economic integration on credit cycles in two subsamples: LMES and HIES}

In this sub-section, we propose the same analysis than the one presented above but for two subsamples: one including 32 LMEs and another sample with 28 HIEs. This section discusses the results for each of these samples.

\section{2.a) Low and Middle income economies}

Table 5 presents the effects of institutions, inward FDI, and trade openness on the Private credit by deposit money banks to GDP (Cre1vo) in the context of LMEs.

Some results are very interesting: control variables including bank capital (Cap), bank return (ROA), bank risk (Risk), real GDP growth (GDPg) confirm previous studies claiming that the banking system with a better capital or profitability would likely increase their credit supply higher while riskier banking systems would reduce their credit supply. Also, our findings confirm that economic growth is one of the main driver of high credit growth in LMEs. 
Institutions, Economic Openness and Credit volatility: Credit Booming and Recession Periods

\begin{tabular}{|c|c|c|c|c|c|c|c|}
\hline Dep. Var: Cre1vo & (1) & (2) & (3) & (4) & (5) & (6) & (7) \\
\hline Indep. Var: & INST & Concor & Goveff & Politic & Requa & Law & Voice \\
\hline \multicolumn{8}{|c|}{ Part A: If Cre1vo> $>0$} \\
\hline \multirow[t]{2}{*}{ Cap(-1) } & 0.009 & 0.008 & 0.008 & 0.010* & 0.009 & 0.008 & $0.011 * *$ \\
\hline & {$[0.006]$} & {$[0.006]$} & {$[0.006]$} & {$[0.006]$} & {$[0.006]$} & {$[0.006]$} & {$[0.006]$} \\
\hline \multirow[t]{2}{*}{ ROA(-1) } & $0.075 * * *$ & $0.068 * * *$ & $0.067 * * *$ & $0.082 * * *$ & $0.077 * * *$ & $0.069 * * *$ & $0.086 * * *$ \\
\hline & {$[0.021]$} & {$[0.021]$} & {$[0.021]$} & {$[0.021]$} & {$[0.021]$} & {$[0.021]$} & {$[0.022]$} \\
\hline \multirow[t]{2}{*}{ Risk(-1) } & $-0.005 * * *$ & $-0.005^{* * *}$ & $-0.005 * * *$ & $-0.005 * * *$ & $-0.005^{* * *}$ & $-0.005 * * *$ & $-0.005 * * *$ \\
\hline & {$[0.002]$} & {$[0.002]$} & {$[0.002]$} & {$[0.002]$} & {$[0.002]$} & {$[0.002]$} & {$[0.002]$} \\
\hline \multirow[t]{2}{*}{ GDPg(-1) } & $0.021 * * *$ & $0.020 * * *$ & $0.020 * * *$ & $0.022 * * *$ & $0.021 * * *$ & $0.020 * * *$ & $0.023 * * *$ \\
\hline & {$[0.005]$} & {$[0.005]$} & {$[0.005]$} & {$[0.005]$} & {$[0.005]$} & {$[0.005]$} & {$[0.005]$} \\
\hline \multirow[t]{2}{*}{ Trade(-1) } & 0.0001 & 0.0002 & 0.0002 & -0.0001 & 0.0001 & 0.0001 & $-8.4 e-06$ \\
\hline & {$[0.0002]$} & {$[0.0002]$} & {$[0.0002]$} & {$[0.0002]$} & {$[0.0002]$} & {$[0.0002]$} & {$[0.0001]$} \\
\hline \multirow[t]{2}{*}{ FDI(-1) } & $0.001 *$ & $0.001 *$ & 0.001 & 0.001 & $0.001 *$ & $0.001 *$ & 0.001 \\
\hline & {$[0.0006]$} & {$[0.0006]$} & {$[0.001]$} & {$[0.001]$} & {$[0.0006]$} & {$[0.0006]$} & {$[0.001]$} \\
\hline \multirow[t]{2}{*}{ INST(-1) } & -0.021 & $-0.037 * *$ & $-0.040 *$ & 0.011 & -0.015 & $-0.039 * *$ & 0.023 \\
\hline & {$[0.022]$} & {$[0.017]$} & {$[0.022]$} & {$[0.018]$} & {$[0.023]$} & {$[0.019]$} & {$[0.019]$} \\
\hline \multirow[t]{2}{*}{ Cons. } & 0.085 & 0.104 & 0.107 & 0.061 & 0.078 & 0.105 & 0.030 \\
\hline & {$[0.067]$} & {$[0.063]$} & {$[0.067]$} & {$[0.055]$} & {$[0.065]$} & {$[0.065]$} & {$[0.069]$} \\
\hline $\mathrm{N}$ & 363 & 363 & 363 & 363 & 363 & 363 & 363 \\
\hline R-squared & 0.264 & 0.272 & 0.270 & 0.263 & 0.263 & 0.271 & 0.266 \\
\hline \multicolumn{8}{|c|}{ Part B: If Cre1vo<0 } \\
\hline \multirow[t]{2}{*}{ Cap(-1) } & $-0.008 *$ & $-0.009 * *$ & -0.006 & $-0.014 * * *$ & $-0.008 *$ & $-0.007 *$ & $-0.011 * *$ \\
\hline & {$[0.004]$} & {$[0.004]$} & {$[0.004]$} & {$[0.004]$} & {$[0.004]$} & {$[0.004]$} & {$[0.004]$} \\
\hline \multirow[t]{2}{*}{ ROA(-1) } & 0.023 & 0.023 & 0.023 & 0.020 & 0.022 & 0.023 & 0.023 \\
\hline & {$[0.016]$} & {$[0.016]$} & {$[0.016]$} & {$[0.016]$} & {$[0.016]$} & {$[0.016]$} & {$[0.016]$} \\
\hline \multirow[t]{2}{*}{ Risk(-1) } & 0.002 & 0.002 & 0.002 & 0.002 & 0.002 & 0.002 & 0.002 \\
\hline & {$[0.002]$} & {$[0.002]$} & {$[0.002]$} & {$[0.002]$} & {$[0.002]$} & {$[0.002]$} & {$[0.002]$} \\
\hline \multirow[t]{2}{*}{ GDPg(-1) } & $0.014 * * *$ & $0.013 * *$ & $0.014 * * *$ & $0.011 * *$ & $0.015 * * *$ & $0.014 * * *$ & $0.013 * *$ \\
\hline & {$[0.005]$} & {$[0.005]$} & {$[0.005]$} & [0.005] & {$[0.005]$} & {$[0.005]$} & {$[0.005]$} \\
\hline \multirow[t]{2}{*}{ Trade(-1) } & -0.0002 & -0.0001 & -0.0003 & 0.00004 & -0.0002 & -0.0002 & 0.0001 \\
\hline & {$[0.0002]$} & {$[0.0002]$} & {$[0.0002]$} & {$[0.0002]$} & {$[0.0002]$} & {$[0.0002]$} & {$[0.0002]$} \\
\hline \multirow[t]{2}{*}{ FDI(-1) } & $-0.003 * *$ & $-0.003 * *$ & $-0.003^{*}$ & $-0.003^{*}$ & $-0.003^{*}$ & $-0.003 *$ & $-0.004 * *$ \\
\hline & {$[0.002]$} & {$[0.002]$} & {$[0.002]$} & {$[0.002]$} & {$[0.002]$} & {$[0.002]$} & {$[0.002]$} \\
\hline \multirow[t]{2}{*}{ INST(-1) } & $0.065 * * *$ & $0.048 * *$ & $0.072 * * *$ & 0.017 & $0.075 * * *$ & $0.060 * * *$ & 0.044* \\
\hline & {$[0.024]$} & {$[0.018]$} & {$[0.021]$} & {$[0.020]$} & {$[0.027]$} & {$[0.020]$} & {$[0.025]$} \\
\hline \multirow[t]{2}{*}{ Cons. } & $-0.340^{* * *}$ & $-0.327 * * *$ & $-0.358^{* * *}$ & $-0.274 * * *$ & $-0.348 * * *$ & $-0.346^{* * *}$ & $-0.329 * * *$ \\
\hline & {$[0.051]$} & {$[0.048]$} & {$[0.053]$} & {$[0.041]$} & {$[0.056]$} & {$[0.052]$} & {$[0.054]$} \\
\hline $\mathrm{N}$ & 357 & 357 & 357 & 357 & 357 & 357 & 357 \\
\hline R-squared & 0.087 & 0.085 & 0.097 & 0.069 & 0.092 & 0.090 & 0.077 \\
\hline No. of countries & 60 & 60 & 60 & 60 & 60 & 60 & 60 \\
\hline
\end{tabular}

Note: Standard errors are in $\left[.{ }^{*}, * *, * * *\right.$ are significant levels at $10 \%, 5 \%, 1 \%$, respectively.

In the case of our explanatory variables, the inward FDI has a positive influence on credit cycles whereas trade openness has a negative one. These results are consistent with previous findings, however, the effect of the economic integration is not always statistical significant meaning that the major drivers of credit growth in the LMEs are still the real GDP growth and the financial system conditions (Auel and de Mendonça, 2011). In addition, the institutions have a positive effect on credit cycles confirming the positive influence of better institutions on the credit growth in the LMEs. Moreover, the results of the logit regression in Part B of the Table 8 shows a significant impact of the trade openness and institutions on the probability of observing credit cycles. The negative effect of trade openness means that a higher trade 
integration reduces the probability of credit booming while better institutions increase the probability of booming credit cycles in the LMEs. This finding can be understood by the fact that better institutions induce booming economic activities in the LMEs with relatively low institutional quality; in such situation, the credit growth would mainly be supported by the economic agents' demand and the policy of the government (Omri et al., 2015). The Table 6 below reports the results for two cases of estimations: the booming period and the recession period in credit markets.

Table 6

Institutions, Economic Openness and Credit volatility - Summary for Robustness check

\begin{tabular}{|c|c|c|c|c|c|c|c|}
\hline \multicolumn{8}{|c|}{ Part A: Robust Pool OLS regression - Cre2vo } \\
\hline Indep. Var: & INST & Concor & Goveff & Politic & Requa & Law & Voice \\
\hline \multirow[t]{2}{*}{ Trade(-1) } & $-0.001 * * *$ & $-0.0006 * * *$ & $-0.001 * * *$ & $-0.0005 * *$ & $-0.001 * * *$ & $-0.0006 * * *$ & $-0.0005^{* *}$ \\
\hline & {$[0.0002]$} & {$[0.0002]$} & {$[0.0002]$} & {$[0.0002]$} & {$[0.0002]$} & {$[0.0002]$} & {$[0.0002]$} \\
\hline \multirow[t]{2}{*}{ FDI(-1) } & $0.002 * *$ & $0.002 * *$ & $0.002 * *$ & $0.002 * *$ & $0.002 * *$ & $0.002 * *$ & $0.002 * *$ \\
\hline & {$[0.001]$} & {$[0.001]$} & {$[0.001]$} & {$[0.001]$} & {$[0.001]$} & {$[0.001]$} & {$[0.001]$} \\
\hline \multirow[t]{2}{*}{ INST(-1) } & $0.055 * *$ & $0.037 * *$ & $0.063 * * *$ & 0.016 & $0.066 * *$ & $0.040 *$ & $0.041^{*}$ \\
\hline & {$[0.024]$} & {$[0.019]$} & {$[0.022]$} & {$[0.019]$} & {$[0.026]$} & {$[0.021]$} & {$[0.022]$} \\
\hline \multicolumn{8}{|c|}{ Part B: Logit regression - DUM2 } \\
\hline \multirow[t]{2}{*}{ Trade(-1) } & $-0.003 * *$ & $-0.003 * *$ & $-0.004 * *$ & -0.002 & $-0.003 * *$ & $-0.003 * *$ & -0.002 \\
\hline & {$[0.002]$} & {$[0.001]$} & {$[0.002]$} & {$[0.002]$} & {$[0.002]$} & {$[0.002]$} & {$[0.001]$} \\
\hline \multirow[t]{2}{*}{ FDI(-1) } & $0.014 * *$ & $0.014 *$ & $0.015 * *$ & $0.015^{*}$ & $0.014 * *$ & $0.014 * *$ & $0.014 *$ \\
\hline & {$[0.007]$} & {$[0.007]$} & {$[0.007]$} & {$[0.007]$} & {$[0.007]$} & {$[0.007]$} & {$[0.007]$} \\
\hline \multirow[t]{2}{*}{ INST(-1) } & 0.212 & $0.203 * *$ & $0.329 * * *$ & 0.030 & 0.206 & 0.182 & 0.046 \\
\hline & {$[0.130]$} & {$[0.101]$} & {$[0.118]$} & {$[0.109]$} & {$[0.131]$} & {$[0.111]$} & {$[0.117]$} \\
\hline \multicolumn{8}{|c|}{ Part C: Robust Pool OLS regression - Cre3vo } \\
\hline \multirow[t]{2}{*}{ Trade(-1) } & $-0.0006 * *$ & $-0.0006 * *$ & $-0.0007 * * *$ & $-0.0005 *$ & $-0.0007 * *$ & $-0.0006 * *$ & $-0.0004 *$ \\
\hline & {$[0.0003]$} & {$[0.0003]$} & {$[0.0003]$} & {$[0.0003]$} & {$[0.0003]$} & {$[0.0003]$} & {$[0.0002]$} \\
\hline \multirow[t]{2}{*}{ FDI(-1) } & $0.002 *$ & $0.002 *$ & $0.002 *$ & $0.002 *$ & $0.002 *$ & $0.002 *$ & 0.002 \\
\hline & {$[0.001]$} & {$[0.001]$} & {$[0.001]$} & {$[0.001]$} & {$[0.001]$} & {$[0.001]$} & {$[0.001]$} \\
\hline \multirow[t]{2}{*}{ INST(-1) } & $0.054 * *$ & $0.037 *$ & $0.063 * *$ & 0.019 & $0.062 * *$ & $0.043 *$ & 0.036 \\
\hline & {$[0.027]$} & {$[0.021]$} & {$[0.025]$} & {$[0.022]$} & {$[0.029]$} & {$[0.024]$} & {$[0.024]$} \\
\hline \multicolumn{8}{|c|}{ Part D: Logit regression - DUM3 } \\
\hline \multirow[t]{2}{*}{ Trade(-1) } & -0.002 & -0.002 & $-0.003 *$ & -0.002 & -0.002 & -0.002 & -0.002 \\
\hline & {$[0.001]$} & {$[0.001]$} & {$[0.001]$} & {$[0.001]$} & {$[0.001]$} & {$[0.001]$} & {$[0.001]$} \\
\hline \multirow[t]{2}{*}{ FDI(-1) } & 0.008 & 0.008 & 0.008 & 0.008 & 0.008 & 0.008 & 0.008 \\
\hline & {$[0.007]$} & {$[0.007]$} & {$[0.007]$} & {$[0.006]$} & {$[0.007]$} & {$[0.007]$} & {$[0.007]$} \\
\hline \multirow[t]{2}{*}{ INST(-1) } & 0.094 & 0.072 & $0.188^{*}$ & 0.028 & 0.102 & 0.051 & 0.010 \\
\hline & {$[0.125]$} & {$[0.098]$} & {$[0.114]$} & {$[0.106]$} & {$[0.127]$} & {$[0.108]$} & {$[0.112]$} \\
\hline \multicolumn{8}{|c|}{ Part E: Robust Pool OLS regression - Cre4vo } \\
\hline \multirow[t]{2}{*}{ Trade(-1) } & $-0.0006 * *$ & $-0.0006 * *$ & $-0.0007 * * *$ & $-0.0005 *$ & $-0.0007 * *$ & $-0.0006 * *$ & $-0.0004 *$ \\
\hline & {$[0.0003]$} & {$[0.0003]$} & {$[0.0003]$} & {$[0.0003]$} & {$[0.0003]$} & {$[0.0003]$} & {$[0.0002]$} \\
\hline \multirow[t]{2}{*}{ FDI(-1) } & $0.002 *$ & $0.002 *$ & $0.002 *$ & $0.002 *$ & $0.002 *$ & $0.002 *$ & $0.002 *$ \\
\hline & {$[0.001]$} & {$[0.001]$} & {$[0.001]$} & {$[0.001]$} & {$[0.001]$} & {$[0.001]$} & {$[0.001]$} \\
\hline \multirow[t]{2}{*}{ INST(-1) } & $0.053 *$ & $0.036^{*}$ & $0.064 * * *$ & 0.015 & $0.063 * *$ & $0.042 *$ & 0.034 \\
\hline & {$[0.027]$} & {$[0.021]$} & {$[0.025]$} & {$[0.022]$} & {$[0.029]$} & {$[0.023]$} & {$[0.024]$} \\
\hline \multicolumn{8}{|c|}{ Part G: Logit regression - DUM4 } \\
\hline \multirow[t]{2}{*}{ Trade(-1) } & $-0.003 *$ & $-0.003 *$ & $-0.003 * *$ & -0.002 & $-0.003 *$ & $-0.003 *$ & -0.002 \\
\hline & {$[0.001]$} & {$[0.001]$} & {$[0.002]$} & {$[0.001]$} & {$[0.001]$} & {$[0.001]$} & {$[0.001]$} \\
\hline \multirow[t]{2}{*}{ FDI(-1) } & 0.008 & 0.008 & 0.008 & 0.008 & 0.008 & 0.008 & 0.008 \\
\hline & {$[0.007]$} & {$[0.007]$} & {$[0.007]$} & {$[0.007]$} & {$[0.007]$} & {$[0.007]$} & {$[0.007]$} \\
\hline \multirow[t]{2}{*}{ INST(-1) } & 0.141 & 0.110 & $0.249 * *$ & 0.020 & 0.159 & 0.093 & 0.049 \\
\hline & {$[0.125]$} & {$[0.098]$} & {$[0.115]$} & {$[0.106]$} & {$[0.128]$} & {$[0.108]$} & {$[0.112]$} \\
\hline
\end{tabular}

Note: Standard errors are in $\left[.{ }^{*}, * *, * * *\right.$ are significant levels at $10 \%, 5 \%, 1 \%$, respectively. 
Results show that institutional indicators (excluding political stability and voice and accountability) have a significant negative effect in the context of positive credit cycles (Cre1vo $>=0$ ); and an insignificant positive effect in a context of negative credit cycles (Cre1vo $<0)$. These results imply a very important observation for the LMEs facing with a high growth rate of financial systems: institutional quality might be an excellent choice to limit the possibility of credit booming in these countries.

Regarding the economic integration, the influence of FDI inflows are the same with our previous results: FDI inflows increase the credit cycles in booming period and they reduce the credit growth in period of recession. However, the trade openness has an opposite effect than what we observe globally. A higher trade openness reduces the credit cycles in booming period while it induces higher credit growth in recession period. This means that the higher trade openness tends to stabilize the credit market by reducing the credit cycles in the LMEs. The results provided in this section suggest that institutional reforms combined with trade openness in the LMEs might be an appropriate policy to stabilize the credit cycles. The robustness check for the case of LMEs with three alternative proxies of credit volatility (Cre2vo, Cre3vo, Cre4vo) shows properly consistent conclusion and it can be provided on request.

\section{2.b) High income economies}

Table 7 hereafter presents the influence of institutions and economic openness on the credit cycles (Cre1vo) for 28 High income economies.

Table 7

Institutions, Economic Openness and Credit volatility: Credit Booming and Recession Periods (Summary for Robustness check)

\begin{tabular}{|c|c|c|c|c|c|c|c|}
\hline Indep. Var: & INST & Concor & Goveff & Politic & Requa & Law & Voice \\
\hline \multicolumn{8}{|c|}{ Part A: If Cre2vo $>=0$} \\
\hline \multirow[t]{2}{*}{ Trade(-1) } & 0.00002 & 0.0001 & 0.0001 & -0.0001 & $-9.3 e-06$ & 0.0001 & -0.00004 \\
\hline & {$[0.0002]$} & {$[0.0002]$} & [0.0002] & {$[0.0002]$} & {$[0.0002]$} & {$[0.0002]$} & [0.0001] \\
\hline \multirow[t]{2}{*}{ FDI(-1) } & $0.001 *$ & $0.001 *$ & 0.001 & 0.001 & 0.001 & $0.001 *$ & 0.001 \\
\hline & {$[0.0006]$} & {$[0.0006]$} & {$[0.001]$} & {$[0.001]$} & {$[0.001]$} & {$[0.0006]$} & {$[0.001]$} \\
\hline \multirow[t]{2}{*}{ INST(-1) } & -0.017 & $-0.035 * *$ & $-0.040 *$ & 0.014 & -0.008 & $-0.036^{*}$ & 0.022 \\
\hline & {$[0.022]$} & {$[0.017]$} & {$[0.022]$} & {$[0.018]$} & {$[0.023]$} & [0.019] & [0.019] \\
\hline \multicolumn{8}{|c|}{ Part B: If $C r e 2 v o<0$} \\
\hline \multirow[t]{2}{*}{ Trade(-1) } & -0.0003 & -0.0003 & $-0.0004 * *$ & -0.0001 & $-0.0004 *$ & $-0.0004 *$ & -0.00006 \\
\hline & [0.0002] & {$[0.0002]$} & {$[0.0002]$} & {$[0.0002]$} & {$[0.0002]$} & {$[0.0002]$} & {$[0.0002]$} \\
\hline \multirow[t]{2}{*}{ FDI(-1) } & $-0.003^{*}$ & $-0.003 *$ & -0.003 & -0.003 & $-0.003 *$ & -0.003 & $-0.003 *$ \\
\hline & {$[0.002]$} & {$[0.002]$} & {$[0.002]$} & {$[0.002]$} & {$[0.002]$} & {$[0.002]$} & {$[0.002]$} \\
\hline \multirow[t]{2}{*}{ INST(-1) } & $0.074 * * *$ & $0.054 * * *$ & $0.075 * * *$ & 0.023 & $0.085 * * *$ & $0.067 * * *$ & $0.052^{* *}$ \\
\hline & [0.024] & {$[0.018]$} & {$[0.021]$} & {$[0.020]$} & {$[0.027]$} & {$[0.020]$} & [0.024] \\
\hline \multicolumn{8}{|c|}{ Part C: If Cre3vo $>=0$} \\
\hline \multirow[t]{2}{*}{ Trade(-1) } & 0.0001 & 0.0001 & 0.0002 & -0.0001 & 0.0001 & 0.0001 & -0.0001 \\
\hline & {$[0.0002]$} & {$[0.0002]$} & {$[0.0002]$} & {$[0.0002]$} & {$[0.0002]$} & {$[0.0002]$} & {$[0.0002]$} \\
\hline \multirow[t]{2}{*}{ FDI(-1) } & $0.001 *$ & $0.001 *$ & 0.001 & 0.001* & $0.001 *$ & $0.001 *$ & $0.001 *$ \\
\hline & [0.0006] & {$[0.0006]$} & {$[0.001]$} & {$[0.0006]$} & {$[0.0006]$} & {$[0.0006]$} & {$[0.0006]$} \\
\hline \multirow[t]{2}{*}{ INST(-1) } & -0.038 & $-0.040 *$ & $-0.063 * *$ & 0.009 & -0.036 & $-0.049 * *$ & 0.002 \\
\hline & {$[0.028]$} & {$[0.022]$} & {$[0.029]$} & {$[0.021]$} & {$[0.029]$} & {$[0.024]$} & {$[0.020]$} \\
\hline \multicolumn{8}{|c|}{ Part D: If $C r e 3 v o<0$} \\
\hline \multirow[t]{2}{*}{ Trade(-1) } & -0.0003 & -0.0003 & $-0.0004 *$ & -0.00001 & -0.0004 & -0.0004 & 0.00004 \\
\hline & {$[0.0002]$} & {$[0.0002]$} & {$[0.0002]$} & {$[0.0002]$} & {$[0.0002]$} & {$[0.0002]$} & {$[0.0002]$} \\
\hline FDI(-1) & -0.002 & -0.002 & -0.002 & -0.001 & -0.002 & -0.002 & -0.002 \\
\hline
\end{tabular}




\begin{tabular}{|c|c|c|c|c|c|c|c|}
\hline & {$[0.002]$} & {$[0.002]$} & {$[0.002]$} & {$[0.002]$} & {$[0.002]$} & {$[0.002]$} & {$[0.002]$} \\
\hline \multirow{2}{*}{ INST(-1) } & $0.097 * * *$ & $0.076 * * *$ & $0.101 * * *$ & 0.021 & $0.108 * * *$ & $0.100 * * *$ & $0.061 * *$ \\
\hline & {$[0.028]$} & [0.021] & {$[0.025]$} & [0.024] & [0.031] & [0.024] & [0.027] \\
\hline \multicolumn{8}{|c|}{ Part E: If Cre4vo $>=0$} \\
\hline \multirow[t]{2}{*}{ Trade(-1) } & 0.0001 & 0.0002 & 0.0002 & -0.0001 & 0.0001 & 0.0002 & -0.00005 \\
\hline & {$[0.0002]$} & {$[0.0002]$} & {$[0.0002]$} & [0.0002] & {$[0.0002]$} & {$[0.0002]$} & {$[0.0002]$} \\
\hline \multirow[t]{2}{*}{ FDI(-1) } & 0.001* & $0.001 *$ & 0.0009 & $0.001 *$ & $0.001 *$ & $0.001 *$ & $0.001 *$ \\
\hline & {$[0.0006]$} & {$[0.0006]$} & {$[0.0006]$} & {$[0.0006]$} & {$[0.0006]$} & {$[0.0006]$} & {$[0.0006]$} \\
\hline \multirow[t]{2}{*}{ INST(-1) } & -0.042 & $-0.044 * *$ & $-0.063 * *$ & 0.004 & -0.038 & $-0.051 * *$ & -0.005 \\
\hline & {$[0.028]$} & {$[0.022]$} & {$[0.028]$} & {$[0.021]$} & {$[0.028]$} & {$[0.023]$} & {$[0.020]$} \\
\hline \multicolumn{8}{|c|}{ Part G: If Cre $4 v o<0$} \\
\hline \multirow[t]{2}{*}{ Trade(-1) } & -0.0003 & -0.0003 & -0.0004 & -0.00002 & -0.0004 & -0.0003 & 0.00004 \\
\hline & {$[0.0002]$} & {$[0.0002]$} & {$[0.0002]$} & {$[0.0002]$} & {$[0.0002]$} & {$[0.0002]$} & {$[0.0002]$} \\
\hline \multirow[t]{2}{*}{ FDI(-1) } & -0.002 & -0.002 & -0.001 & -0.001 & -0.002 & -0.002 & -0.002 \\
\hline & {$[0.002]$} & {$[0.002]$} & {$[0.002]$} & {$[0.002]$} & {$[0.002]$} & {$[0.002]$} & {$[0.002]$} \\
\hline \multirow[t]{2}{*}{ INST(-1) } & $0.087 * * *$ & $0.068 * * *$ & $0.091 * * *$ & 0.020 & $0.099 * * *$ & $0.091 * * *$ & $0.052 * *$ \\
\hline & {$[0.027]$} & {$[0.020]$} & {$[0.025]$} & {$[0.023]$} & {$[0.031]$} & {$[0.023]$} & {$[0.026]$} \\
\hline
\end{tabular}

Note: Standard errors are in [. *, **, *** are significant levels at $10 \%, 5 \%, 1 \%$, respectively.

Regarding our control variables, all results are substantially consistent excepting for the case of bank capita. In the HIEs, the banking system with a highly capitalized market have a lower credit growth meaning that the banking system with lower capitalized profile provide higher credit growth through the implementation of risk taking activities in banking system in HIEs.

The results for our explanatory variables indicate that an improvement in institutions have a positive but insignificant effect on credit cycles. Meanwhile, the trade openness has a negative influence whereas the FDI inflows have a positive one. Interestingly, the results of our logit regression in the Part B of the Table 7 show that the trade openness has a significant negative effect and the FDI inflows has a significant positive influence. This means that the economic integration has an opposite effect on credit cycles: the trade openness reduces the credit growth far away from booming, while the FDI inflows support the booming credit growth in the HIEs. These observations highlight the importance of economic integration in explaining the credit cycles in the HIEs where an already well-developped institutional framework play a less important role in the credit market. Table 8 shows the results for estimations in cases of positive credit cycles and negative credit cycles.

Table 8

Institutions, Economic Openness and Credit volatility: Low and Middle Income Economies

\begin{tabular}{|c|c|c|c|c|c|c|c|}
\hline \multicolumn{8}{|c|}{ Part A: Robust Pool OLS regression } \\
\hline Dep. Var: Cre1vo & (1) & (2) & (3) & (4) & (5) & (6) & (7) \\
\hline Indep. Var: & INST & Concor & Goveff & Politic & Requa & Law & Voice \\
\hline \multirow[t]{2}{*}{ Cap(-1) } & $0.012 *$ & $0.012 *$ & $0.013^{*}$ & $0.013 *$ & $0.012 *$ & $0.013 *$ & $0.013 *$ \\
\hline & [0.007] & {$[0.007]$} & [0.007] & {$[0.007]$} & {$[0.007]$} & {$[0.007]$} & [0.007] \\
\hline \multirow[t]{2}{*}{ ROA(-1) } & $0.110 * * *$ & $0.110^{* * *}$ & $0.110^{* * *}$ & $0.108 * * *$ & $0.109 * * *$ & $0.109 * * *$ & $0.109 * * *$ \\
\hline & [0.031] & {$[0.031]$} & {$[0.031]$} & [0.031] & [0.031] & [0.031] & [0.031] \\
\hline \multirow[t]{2}{*}{ Risk(-1) } & $-0.005^{* *}$ & $-0.005^{* *}$ & $-0.005^{* *}$ & $-0.005^{*}$ & $-0.005^{* *}$ & $-0.004 *$ & $-0.004 *$ \\
\hline & [0.002] & {$[0.002]$} & {$[0.002]$} & {$[0.003]$} & [0.003] & {$[0.002]$} & [0.002] \\
\hline \multirow[t]{2}{*}{ GDPg(-1) } & $0.045^{* * *}$ & $0.044 * * *$ & $0.044 * * *$ & $0.045 * * *$ & $0.045 * * *$ & $0.045 * * *$ & $0.046 * * *$ \\
\hline & {$[0.009]$} & [0.009] & {$[0.009]$} & [0.009] & [0.009] & {$[0.009]$} & {$[0.009]$} \\
\hline \multirow[t]{2}{*}{ Trade(-1) } & $-0.001 *$ & -0.001 & -0.001 & -0.001 & -0.001 & -0.001 & -0.001 \\
\hline & {$[0.0007]$} & {$[0.001]$} & [0.001] & [0.001] & {$[0.001]$} & {$[0.001]$} & [0.001] \\
\hline
\end{tabular}




\begin{tabular}{|c|c|c|c|c|c|c|c|}
\hline FDI(-1) & 0.008 & 0.008 & $0.009 *$ & 0.008 & 0.008 & 0.008* & 0.008 \\
\hline & {$[0.005]$} & {$[0.005]$} & {$[0.005]$} & {$[0.005]$} & {$[0.005]$} & {$[0.005]$} & {$[0.005]$} \\
\hline \multirow[t]{2}{*}{ INST(-1) } & 0.074 & 0.047 & 0.040 & 0.027 & 0.053 & -0.004 & 0.043 \\
\hline & {$[0.068]$} & {$[0.057]$} & {$[0.060]$} & {$[0.034]$} & {$[0.057]$} & {$[0.051]$} & {$[0.041]$} \\
\hline \multirow[t]{2}{*}{ Cons. } & $-0.383^{* * *}$ & $-0.397 * * *$ & $-0.409 * * *$ & $-0.406^{* * *}$ & $-0.406^{* * *}$ & $-0.447 * * *$ & $-0.444 * * *$ \\
\hline & {$[0.125]$} & {$[0.126]$} & {$[0.124]$} & {$[0.126]$} & {$[0.119]$} & {$[0.123]$} & {$[0.112]$} \\
\hline $\mathrm{N}$ & 384 & 384 & 384 & 384 & 384 & 384 & 384 \\
\hline R-squared & 0.251 & 0.250 & 0.250 & 0.250 & 0.251 & 0.249 & 0.251 \\
\hline \multicolumn{8}{|c|}{ Part B: Logit regression } \\
\hline Dep. Var: DUM1 & $(1)$ & $(2)$ & (3) & (4) & $(5)$ & (6) & $(7)$ \\
\hline Indep. Var: & INST & Concor & Goveff & Politic & Requa & Law & Voice \\
\hline \multirow[t]{2}{*}{ Cap(-1) } & $0.060 *$ & 0.055 & 0.056 & $0.067 * *$ & $0.060 *$ & 0.064* & $0.068 * *$ \\
\hline & {$[0.034]$} & {$[0.035]$} & {$[0.034]$} & {$[0.034]$} & {$[0.034]$} & {$[0.034]$} & {$[0.034]$} \\
\hline \multirow[t]{2}{*}{ ROA(-1) } & $0.468 * * *$ & $0.477 * * *$ & $0.504 * * *$ & $0.459 * * *$ & $0.460 * * *$ & $0.473 * * *$ & $0.465 * * *$ \\
\hline & [0.179] & {$[0.180]$} & {$[0.190]$} & {$[0.176]$} & {$[0.178]$} & {$[0.179]$} & {$[0.176]$} \\
\hline \multirow[t]{2}{*}{ Risk(-1) } & -0.019 & -0.020 & -0.021 & -0.017 & -0.018 & -0.016 & -0.014 \\
\hline & {$[0.014]$} & {$[0.014]$} & {$[0.014]$} & {$[0.014]$} & {$[0.014]$} & {$[0.014]$} & {$[0.014]$} \\
\hline \multirow[t]{2}{*}{ GDPg(-1) } & $0.198 * * *$ & $0.189 * * *$ & $0.181 * * *$ & $0.195 * * *$ & $0.197 * * *$ & $0.189 * * *$ & $0.192 * * *$ \\
\hline & [0.052] & {$[0.051]$} & {$[0.051]$} & {$[0.052]$} & {$[0.052]$} & {$[0.051]$} & {$[0.054]$} \\
\hline \multirow[t]{2}{*}{ Trade(-1) } & $-0.009 * *$ & $-0.008 * *$ & $-0.011 * * *$ & $-0.007^{*}$ & $-0.008 * *$ & $-0.008 * *$ & $-0.006 *$ \\
\hline & {$[0.004]$} & {$[0.004]$} & {$[0.004]$} & {$[0.004]$} & {$[0.004]$} & {$[0.004]$} & {$[0.004]$} \\
\hline \multirow[t]{2}{*}{ FDI(-1) } & 0.005 & 0.006 & 0.019 & 0.007 & 0.008 & 0.010 & 0.011 \\
\hline & [0.023] & {$[0.023]$} & {$[0.023]$} & [0.024] & {$[0.023]$} & {$[0.023]$} & {$[0.025]$} \\
\hline \multirow[t]{2}{*}{ INST(-1) } & $0.610^{*}$ & $0.594 * *$ & $0.776 * * *$ & 0.150 & 0.345 & 0.298 & 0.004 \\
\hline & {$[0.342]$} & {$[0.285]$} & {$[0.294]$} & {$[0.178]$} & {$[0.275]$} & {$[0.274]$} & {$[0.214]$} \\
\hline \multirow[t]{2}{*}{ Cons. } & $-1.322^{* *}$ & $-1.204^{*}$ & $-1.178^{*}$ & $-1.623 * * *$ & $-1.564 * * *$ & $-1.517 * *$ & $-1.827 * * *$ \\
\hline & {$[0.607]$} & {$[0.623]$} & {$[0.614]$} & {$[0.600]$} & {$[0.583]$} & {$[0.613]$} & {$[0.564]$} \\
\hline $\mathrm{N}$ & 384 & 384 & 384 & 384 & 384 & 384 & 384 \\
\hline No. of countries & 32 & 32 & 32 & 32 & 32 & 32 & 32 \\
\hline
\end{tabular}

Note: Standard errors are in $\left[.{ }^{*},{ }^{*},{ }^{* * *}\right.$ are significant levels at $10 \%, 5 \%, 1 \%$, respectively.

The results above show that the economic integration has a positive effect on credit cycles in booming period, while it has a negative effect on these cycles in recession period. In other words, economic integration generates stronger credit cycles. In contrast, the institutions have an opposite effect on credit cycles. For robustness purpose, this analysis has been consistently done with three different proxies of credit levels (Cre2vo, Cre3vo, Cre4vo) confirming our result - this robustness check can be provided on request.

\section{CONCLUSION}

This article investigates the influence of institutions, foreign direct investment and trade openness on the credit cycles in 60 economies (categorized into two sub-samples: LMEs and HIES). The contribution of this study is to investigate the combined effect of institutions and economic integration for the different credit cycles of a global sample over the period of 2003-2017.

Generally speaking, our results show that, the effect of inward FDI on the credit cycles is positive while the influence of trade openness is significantly negative. The credit cycles are also affected by institutional quality. Precisely, better institutions induce a higher growth rate of credit levels which then induce credit cycles toward booming period. Meanwhile, the economic integration has an opposite effect on credit cycles on two aspects: trade openness and FDI inflows. The trade openness reduces the credit growth while FDI inflows induce a credit growth toward booming period. 
The trade openness and FDI inflows have a positive effect on the credit growth during a booming period while they have a negative influence in recession period. This means that a higher economic integration exacerbates the dynamics of credits. Notably, the institutions exhibit a negative influence on the credit growth in credit booming period while it has a positive effect in credit recession period. This observation shows the important roles of institutions in curving the credit cycles and helping to reduce the effects of economic integration on credit cycles.

Thirdly, the effects of institutions and economic integration on credit cycles are confirmed for both LMEs and HIEs. For LMEs, the economic integration plays a more important role (than institutions) in affecting credit cycles. Interestingly, the trade openness and institutions help to stabilize credit cycles. In HIEs, the economic integration exacerbates credit cycles while institutions marginally help to stabilize them.

\section{ACKNOWLEDGEMENT}

This study is funded by the University of Economics Ho Chi Minh City, Vietnam

\section{REFERENCES}

Acemoglu, D. \& Robinson, J. 2008. The role of institutions in growth and development. World Bank, Washington DC.

Aikman, D., Haldane, A. G. \& Nelson, B. D. 2015. Curbing the credit cycle. The Economic Journal, 125, 10721109.

Altunbaş, Y., Fazylov, O. \& Molyneux, P. 2002. Evidence on the bank lending channel in Europe. Journal of Banking \& Finance, 26, 2093-2110.

Altunbas, Y., Gambacorta, L. \& Marques-Ibanez, D. 2010. Bank risk and monetary policy. Journal of Financial Stability, 6, 121-129.

Apostoaie, C.-M. \& Percic, S. 2014. Credit Cycles and Business Cycles in Twenty EU Economies. Procedia Economics and Finance, 15, 1055-1064.

Araujo, L., Mion, G. \& Ornelas, E. 2016. Institutions and export dynamics. Journal of International Economics, 98, 2-20.

Auel, M. C. \& De Mendonça, H. F. 2011. Macroeconomic relevance of credit channels: Evidence from an emerging economy under inflation targeting. Economic Modelling, 28, 965-979.

Aysun, U. \& Hepp, R. 2013. Identifying the balance sheet and the lending channels of monetary transmission: A loan-level analysis. Journal of Banking \& Finance, 37, 2812-2822.

Backé, P. \& Wójcik, C. 2008. Credit booms, monetary integration and the new neoclassical synthesis. Journal of Banking \& Finance, 32, 458-470.

Bernanke, B. S. \& Gertler, M. 1995. Inside the black box: the credit channel of monetary policy transmission. Journal of Economic perspectives, 9, 27-48.

Bucsa G., Jovanovic J. and Schinckus C. 2011. A unified econophysics for price return distributions. Physica A, 390, 3435-3443.

Canh, N. P. 2016. Monetary policy transmission and bank lending channel in Vietnam. Trư ờ ng Đạ i họ c Kinh tế Tp. Hồ Chí Minh.

Canh, N. P., Schinckus, C. \& Thanh, S. D. 2018a. Do economic openness and institutional quality influence patents? Evidence from GMM systems estimates. International Economics, https://doi.org/10.1016/j.inteco.2018.10.

Canh, N. P., Thai, N. V. H. \& Schinckus, C. 2018b. Stock-Return Co-Movements and Institutional Quality: An Empirical Investigation of the European Emerging Markets. Theoretical economics letters, 8, 820843.

Chen, X., Kontonikas, A. \& Montagnoli, A. 2012. Asset prices, credit and the business cycle. Economics Letters, 117, 857-861.

De Moraes, C. O., Montes, G. C. \& Antunes, J. A. P. 2016. How does capital regulation react to monetary policy? New evidence on the risk-taking channel. Economic Modelling, 56, 177-186. 
Dees, S. 2016. Credit, asset prices and business cycles at the global level. Economic Modelling, 54, 139-152.

Dell' ariccia, G., Laeven, L. \& Marquez, R. 2014. Real interest rates, leverage, and bank risk-taking. Journal of Economic Theory, 149, 65-99.

Dumitrescu, E.-I. \& Hurlin, C. 2012. Testing for Granger non-causality in heterogeneous panels. Economic Modelling, 29, 1450-1460.

Duprey, T. 2012. Bank Ownership and Credit Cycle: the lower sensitivity of public bank lending to the business cycle.

Eickmeier, S. \& NG, T. 2015. How do US credit supply shocks propagate internationally? A GVAR approach. European Economic Review, 74, 128-145.

Herrera-Echeverri, H., Haar, J. \& Estévez-Bretón, J. B. 2014. Foreign direct investment, institutional quality, economic freedom and entrepreneurship in emerging markets. Journal of Business Research, 67, 19211932.

Huynh, C. M., Nguyen, V. H. T., Nguyen, H. B. \& Nguyen, P. C. 2020. One-way effect or multiple-way causality: foreign direct investment, institutional quality and shadow economy? International Economics and Economic Policy, 17, 219-239.

Igan, D. \& Tan, Z. 2015. Capital Inflows, Credit Growth, and Financial Systems, International Monetary Fund.

Imran, K. \& Nishat, M. 2013. Determinants of bank credit in Pakistan: A supply side approach. Economic Modelling, 35, 384-390.

Jiménez, G., Saurina, J. \& De España, B. 2005. Credit cycles, credit risk and prudential regulation. Banco de España.

Kaminsky, G. L. \& Reinhart, C. M. 1999. The twin crises: the causes of banking and balance-of-payments problems. American economic review, 473-500.

Karfakis, C. 2013. Credit and business cycles in Greece: Is there any relationship? Economic Modelling, 32, 2329.

Kiss, G., Nagy, M. \& Vonnák, B. Credit growth in central and eastern europe: trend, cycle or boom. conference "Finance and Consumption Workshop: Consumption and Credit in Countries with Developing Credit Markets", Florence, 2006. 16-17.

Krugman, P. 1999. What happened to Asia, Springer.

Lambertini, L., Mendicino, C. \& Teresa Punzi, M. 2013. Leaning against boom-bust cycles in credit and housing prices. Journal of Economic Dynamics and Control, 37, 1500-1522.

Luo Z. and Schinckus C. 2015. Herding Behaviour in asymmetric and extreme situations: the case of China. Applied Economics Letters, Vol. 22 (11), 869-873.

Magud, N. E. \& Vesperoni, E. R. 2015. Exchange rate flexibility and credit during capital inflow reversals: Purgatory ... not paradise. Journal of International Money and Finance, 55, 88-110.

Matsuyama, K., Sushko, I. \& Gardini, L. 2016. Revisiting the model of credit cycles with Good and Bad projects. Journal of Economic Theory, 163, 525-556.

Mendoza, E. G. \& Terrones, M. E. 2008. An anatomy of credit booms: evidence from macro aggregates and micro data. National Bureau of Economic Research.

Nguyen, C. P., Nguyen, N. A., Schinckus, C. \& SU, T. D. 2018a. The ambivalent role of institutions in the CO2 emissions: The case of emerging countries. International Journal of Energy Economics and Policy, 8,7 .

Nguyen, C. P., SU, T. D. \& Nguyen, T. V. H. 2018b. Institutional Quality and Economic Growth: The Case of Emerging Economies. Theoretical Economics Letters, 8, 1943.

Nguyen, C. P., Schinckus C., SU T. and Chong F.H.L. 2018c. Institutions, Inward Foreign Direct Investment, Trade Openness and Credit Level in Emerging Economies. Review of Development Finance, vol. 8 (2), p. 75-88.

Omri, A., Daly, S., Rault, C. \& Chaibi, A. 2015. Financial development, environmental quality, trade and economic growth: What causes what in MENA countries. Energy Economics, 48, 242-252.

Petacchi, R. 2015. Information asymmetry and capital structure: Evidence from regulation FD. Journal of Accounting and Economics, 59, 143-162.

Phuc Nguyen, C., Schinckus, C., Dinh SU, T. \& Chong, F. 2018. Institutions, inward foreign direct investment, trade openness and credit level in emerging market economies. Review of Development Finance, 8, 75-88. 
Ramos-Tallada, J. 2015. Bank risks, monetary shocks and the credit channel in Brazil: Identification and evidence from panel data. Journal of International Money and Finance, 55, 135-161.

Rubaszek, M. \& Serwa, D. 2014. Determinants of credit to households: An approach using the life-cycle model. Economic Systems, 38, 572-587.

Samarina, A. \& Bezemer, D. 2016. Do capital flows change domestic credit allocation? Journal of International Money and Finance, 62, 98-121.

Shen, C.-H., Lee, Y. H., Wu, M.-W. \& Guo, N. 2016. Does housing boom lead to credit boom or is it the other way around? The case of China. International Review of Economics \& Finance, 42, 349-367.

Thong, N. T. \& Canh, N. P. Institution, external debts, and fiscal policy: an empirical investigation in Asia Pacific countries. Proceedings of ICUEH2016: International conference of University of Economic Ho Chi Minh City: Policies and sustainable economic development, HCMC, Vietnam, November 11, 2016, 2016. UEH Publishing House, 528-549.

Vo, X. V. \& Nguyen, P. C. 2014. Monetary Policy and Bank Credit Risk in Vietnam Pre and Post Global Financial Crisis. Contemporary Studies in Economic and Financial Analysis, 96, 277-290. 